\title{
Deuteronlike Heavy Dibaryons from Lattice Quantum Chromodynamics
}

\author{
Parikshit Junnarkar@* and Nilmani Mathur \\ Department of Theoretical Physics, Tata Institute of Fundamental Research, Homi Bhabha Road, Mumbai 400005, India
}

(Received 1 July 2019; revised manuscript received 16 August 2019; published 18 October 2019)

\begin{abstract}
We report the first lattice quantum chromodynamics (QCD) study of deuteronlike ( $n p$-like) dibaryons with heavy quark flavors. These include particles with the following dibaryon structures and valence quark contents: $\Sigma_{c} \Xi_{c c}(u u c u c c), \Omega_{c} \Omega_{c c}(\operatorname{sscsc}), \Sigma_{b} \Xi_{b b}(u u b u b b), \Omega_{b} \Omega_{b b}(\operatorname{ssb} b b)$, and $\Omega_{c c b} \Omega_{c b b}(c c b c b b)$, and with spin $(J)$ parity $(P), J^{P} \equiv 1^{+}$. Using a state-of-the art lattice QCD calculation, after controlling relevant systematic errors, we unambiguously find that the ground state masses of dibaryons $\Omega_{c} \Omega_{c c}(\operatorname{sscsc} c), \Omega_{b} \Omega_{b b}(\operatorname{ssbsbb})$, and $\Omega_{c c b} \Omega_{c b b}(c c b c b b)$ are below their respective two-baryon thresholds, suggesting the presence of bound states that are stable under strong and electromagnetic interactions. We also predict their masses precisely. For dibaryons $\Sigma_{c} \Xi_{c c}(u u c u c c)$, and $\Sigma_{b} \Xi_{b b}(u u b u b b)$, we could not reach to a definitive conclusion about the presence of any bound state due to large systematics associated with these states. We also find that the binding of these dibaryons becomes stronger as they become heavier in mass. This study also opens up the possibility of the existence of many other exotic nuclei, which can be formed through the fusion of heavy baryons, similar to the formation of nuclei of elements in the periodic table.
\end{abstract}

DOI: $10.1103 /$ PhysRevLett.123.162003

A deuteron is a bound state of two baryons, a proton and a neutron, and is made of six light valence quarks. In the early Universe, deuterons were created and their stability is responsible for the creation of other elements. Interestingly, the strong interactions between quarks, which bring stability to deuterons, also allow various other six-quark combinations leading to the possible formation of many other dibaryons. However, no such strong-interactionstable dibaryons, though speculated about many times [1-8], have been observed yet [9]. Using a state-of-theart first principles calculation of lattice quantum chromodynamics (QCD), here we report, for the first time, a definite prediction of the existence of other deuteronlike spin-1 dibaryons. We also predict their masses precisely. These new subatomic particles could either be made of six heavy quarks (charm and bottom) or heavy and strange quarks. Such dibaryons are stable against strong and electromagnetic decays, but, unlike the deuteron, they can decay through weak interactions. We also find that such dibaryons become more strong-interaction stable as they become heavier. We expect that prediction from this calculation will initiate more theoretical works on heavy dibaryons, particularly to understand their binding mechanism, and may as well aid in discovering these new

Published by the American Physical Society under the terms of the Creative Commons Attribution 4.0 International license. Further distribution of this work must maintain attribution to the author(s) and the published article's title, journal citation, and DOI. Funded by SCOAP. subatomic particles at future experimental facilities, such as at the upgraded Large Hadron Collider and future high energy heavy ion facilities. In fact this study opens up the possibility of the existence of many other exotic nuclei, which can be formed through the fusion of heavy baryons, similar to the formation of nuclei of elements in the periodic table. Formation of these hadrons perhaps also enhances the possibility of a quark-level analog of nuclear fusion as discussed recently [10]. Further study of these exotic states can also provide information on the strong interaction dynamics at multiple scales.

The particular dibaryons $(\mathcal{D})$ [We identify the two-flavor spin-1 dibaryons with the symbol $\mathcal{D}$ and name them as $\mathcal{D}_{q_{1} q_{2}}$, which are made of two baryons with valence quark contents $\left(q_{1} q_{1} q_{2}\right)$ and $\left(q_{1} q_{2} q_{2}\right)$. In this notation the deuteron is $\mathcal{D}_{u d} \equiv n p($ uududd $)$. Such dibaryons may be called as $\mathcal{D}$ dibaryons.], which we investigate, are heavy quark analogs of deuteron $(n p)$. They have the spin- $(J)$ parity $(P)$ quantum numbers: $J^{P}=1^{+}$, with the following dibaryon configurations: $\mathcal{D}_{c u} \equiv \Sigma_{c} \Xi_{c c}($ uucucc $), \mathcal{D}_{c s} \equiv \Omega_{c} \Omega_{c c}(\operatorname{sscscc}), \mathcal{D}_{b u} \equiv$ $\Sigma_{b} \Xi_{b b}\left(\right.$ uububb), $\quad \mathcal{D}_{b s} \equiv \Omega_{b} \Omega_{b b}\left(\right.$ ssbsbb), and $\quad \mathcal{D}_{b c} \equiv$ $\Omega_{c c b} \Omega_{c b b}(c c b c b b)$. Here, $\Sigma_{q}, \Xi_{q q}, \Omega_{q q}, \Omega_{q_{1} q_{2} q_{2}}$ 's are heavy baryons with the usual nomenclature of the Particle Data Group [11], and $u, s, c, b$ inside brackets are various quark flavors. We find that $D_{c s}, D_{b s}$, and $D_{b c}$ are stable against strong and electromagnetic decays, and thus it will be interesting to carry out further theoretical studies on their binding mechanisms as well as their production mechanisms. However, for $\mathcal{D}_{c u}$ and $\mathcal{D}_{b u}$, we find the ground state masses are consistent with their respective two-baryon thresholds, and therefore our results are not currently precise enough to 
reach a definitive conclusion on their stability. Incidentally, only recently tetra- $[12-14]$ and pentaquark $[15,16]$ states have been discovered and those are made of heavy quarks. Recent model and lattice QCD studies also suggest the existence of other heavy tetraquark hadrons [17-23]. It is thus natural to search for six-quark states made of heavy quarks and our predictions provide a first indication for the existence of such heavy hadrons. Dibaryons have been studied through various models over the years [1-8], However, it is quite crucial to have first principles lattice QCD based studies on these states to predict their masses and to understand their structures. In fact, a few lattice QCD studies have already been carried out [24-30]. However, those are mainly focused on light quarks with spin-0 states $[24,25,28,29]$, studies of deuteron in Refs. [26,27], as well as studies of spin-2 states [30]. This work is the first lattice study on heavy dibaryons. A lattice dibaryon calculation with heavy quarks is advantageous over the light counterparts in two ways. The two-point correlators are less noisy in comparison with light dibaryons and, secondly, the signalto-noise ratio of the two-point correlators is far better in comparison to the light quark calculations. Both of these provide an added advantage in our calculation and help in reliable extraction of binding energies of these heavy dibaryons.

The lattice setup that we use here is similar to what was used in Refs. [21,31-33]. Below we elaborate it further for the sake of completeness.

A. Lattice ensembles. - Three sets of dynamical $2+1+1$ flavors $(u / d, s, c)$ lattice ensembles, within a volume of about $3 \mathrm{fm}$, generated by the MILC Collaboration [34] with HISQ fermion action [35], are employed for this study. Lattice spacings, using the $r_{1}$ parameter, for these ensembles are measured to be $0.1207(11), 0.0888(8)$, and $0.0582(5) \mathrm{fm}$, respectively [34], which are also found to be consistent with scales obtained through Wilson flow [36].

B. Quark actions. - In the valence sector, from light to charm quarks, we utilize the overlap action which has exact chiral symmetry at finite lattice spacings [37-39] and no $\mathcal{O}^{n}(m a), n=1,3,5, \cdots$ errors. A nonrelativistic QCD (NRQCD) formulation [40] is adapted for the bottom quark with an $\mathcal{O}\left(\alpha_{s} v^{4}\right)$ improved Hamiltonian with nonperturbatively tuned improvement coefficients [41]. For reliable extraction of the ground states we employ a wall source and point sink.

C. Quark mass tuning.-We tune both the charm and bottom quark masses using the Fermilab prescription for heavy quarks [42] in which we equate the lattice-extracted spin-averaged kinetic masses of the $1 S$ quarkonia states with their physical values [11]. Following Ref. [43] we tune the strange quark mass to its physical value.

D. Dibaryon interpolators. - The dibaryon interpolating operator with spin $(J=1)$ and antisymmetric in flavors $(q, Q) \in(s, c, b)$ is constructed as

$$
\mathcal{D}_{q Q}=\frac{1}{\sqrt{2}}\left(\Omega_{q q Q}\left(C \gamma^{j}\right) \Omega_{Q Q q}-\Omega_{Q Q q}\left(C \gamma^{j}\right) \Omega_{q q Q}\right),
$$

TABLE I. Structures of spin-1 heavy dibaryons that we study in this work.

\begin{tabular}{lc}
\hline \hline $\mathcal{D}_{Q q}$ & Interpolating fields \\
\hline $\mathcal{D}_{b c}$ & $\frac{1}{\sqrt{2}}\left(\Omega_{c c b} \Omega_{b b c}-\Omega_{b b c} \Omega_{c c b}\right)$ \\
$\mathcal{D}_{b s}$ & $\frac{1}{\sqrt{2}}\left(\Omega_{b} \Omega_{b b}-\Omega_{b b} \Omega_{b}\right)$ \\
$\mathcal{D}_{c s}$ & $\frac{1}{\sqrt{2}}\left(\Omega_{c} \Omega_{c c}-\Omega_{c c} \Omega_{c}\right)$ \\
$\mathcal{D}_{b u}$ & $\frac{1}{\sqrt{2}}\left(\Sigma_{b} \Xi_{b b}-\Xi_{b b} \Sigma_{b}\right)$ \\
$\mathcal{D}_{c u}$ & $\frac{1}{\sqrt{2}}\left(\Sigma_{c} \Xi_{c c}-\Xi_{c c} \Sigma_{c}\right)$ \\
\hline \hline
\end{tabular}

where $\Omega_{q q Q}$ and $\Omega_{Q Q q}$ are spin-1/2 baryons defined as $\left(\Omega_{q q Q}\right)_{\alpha}=\epsilon^{a b c} q_{\alpha}^{a}(x) q_{\mu}^{b}(x)\left(C \gamma_{5}\right)_{\mu \nu} Q_{\nu}^{c}(x)$ and $\left(\Omega_{Q Q q}\right)_{\alpha}=$ $\epsilon^{a b c} Q_{\alpha}^{a}(x) q_{\mu}^{b}(x)\left(C \gamma_{5}\right)_{\mu \nu} Q_{\nu}^{c}(x)$. Here Latin letters indicate color, while Greek letters indicate the spinor degree of freedom. The various deuteron analogs with appropriate flavor antisymmetry are listed in Table I.

In a lattice QCD formulation [44], the mass $(m)$ of a particle is extracted in two steps: first by calculating the two-point Euclidean time $(\tau)$ correlator $(\langle C(\tau)\rangle)$ of the interpolating source and sink operators, over many gauge configurations, and then extracting the exponent $(m)$ by fitting the exponential decay $\left(\langle C(\tau)\rangle \sim e^{-m \tau}\right)$ of that correlator at large Euclidean time. Following the dibaryon structure of deuteron $[d=(1 / \sqrt{2})(p n-n p)]$, the spin-1 flavor-antisymmetric dibaryon interpolating fields are constructed with the appropriate spin projection of two individual spin- $1 / 2$ baryons, as shown in Table I. We then calculate the two-point correlators with those interpolating fields and from the exponential decay of these correlators we calculate the lowest energy states, i.e., the ground state masses of each dibaryon. Masses of individual baryons are also calculated similarly (See Supplemental Material [45] for more details on the calculation procedure and error analysis).

The next step is to find the relative energy levels of the ground state dibaryons with respect to their two-baryon thresholds. Since baryon number is a conserved quantity in the standard model of particle physics, these spin-1 dibaryons can have only two strong decay channels: either to two spin-1/2 baryons or to two spin-3/2 baryons. Interestingly, we find that the ordering of masses for these two combinations is different with the charm and the bottom quarks. While the sum of masses of two spin$1 / 2$ charmed baryons $\left(\Sigma_{c}\right.$ and $\Xi_{c c}$ or $\Omega_{c}$ and $\left.\Omega_{c c}\right)$ are smaller than that of two spin-3/2 charmed baryons $\left(\Omega_{q q q, q=u, s}\right.$ and $\left.\Omega_{c c c}\right)$, for the bottom quark this trend is opposite, i.e., $M_{\Sigma_{b}}+M_{\Xi_{b b}}>M_{\Delta_{\text {иии }}}+M_{\Omega_{b b b}}, M_{\Omega_{b}}+M_{\Omega_{b b}}>$ $M_{\Omega_{s s s}}+M_{\Omega_{b b b}}$, and $M_{\Omega_{c c b}}+M_{\Omega_{c b b}}>M_{\Omega_{c c c}}+M_{\Omega_{b b b}}$. These observations are consistent with known experimental results and lattice determination of baryon masses $[32,33,47]$. After computing the dibaryon masses we then calculate their mass differences from both the spin- $1 / 2$ 
and spin-3/2 two-baryon thresholds. When the mass difference of a dibaryon from its closest threshold is negative and the finite volume effects are convincingly small, that particular dibaryon is likely to be a bound state.

We compute the aforementioned mass differences at multiple lattice spacings within our lattice setup. Our results are shown in Fig. 1. Mass differences from spin$1 / 2$ and spin-3/2 thresholds are shown by red and green colors, respectively. The upper plot is for the dibaryon $\mathcal{D}_{b c}\left(\Omega_{c c b} \Omega_{c b b}\right)$, the middle one is for $\mathcal{D}_{b s}\left(\Omega_{s s b} \Omega_{s b b}\right)$, and the bottom one is for $\mathcal{D}_{c s}\left(\Omega_{s s c} \Omega_{s c c}\right)$. It should be noted that these dibaryon energy levels are already computed at their physical quark masses and therefore only require a continuum extrapolation and finite volume corrections. For the continuum extrapolation, the energy levels are computed at three lattice spacings, indicated by different marker styles, and we use a linear in $a^{2}$ ansatz as well as with an $a^{2} \ln (a)$ term. Note that use of overlap action, which has exact chiral symmetry on lattice, ensures no $\mathcal{O}^{n}(m a), n=1,3,5, \cdots$ errors, which then assures that higher order discretization errors are smaller, particularly at the finest lattice. In addition, since we calculate mass splittings, rather than masses, errors due to finite lattices are in good control. The errors shown include both statistical as well as systematic errors, which are then added in quadrature to get the final errors (see Supplemental Material [45] for details on error analysis).

From these figures it is quite apparent that the ground state masses of $\mathcal{D}_{b c}, \mathcal{D}_{b s}$, and $\mathcal{D}_{c s}$ lie below their respective closest two baryon thresholds by about 4,2 , and 3 sigma errors, respectively. Next, it is natural to ask if these mass differences obtained at finite volume lattices $\left(L^{3}\right)$ are indeed the physical binding energies that hold these dibaryons together from decaying to individual baryons. To sort this out one needs to study finite volume corrections of these lattice computed energy levels. Fortunately for multihadron systems this has already been worked out [54-56] and the finite volume corrections, $\Delta_{\mathrm{FV}}$, for such systems was found to be $\sim \mathcal{O}\left(e^{-k_{\infty} L}\right) / L$, where $k_{\infty}=$ $\sqrt{\left(m_{1}+m_{2}\right) B_{\infty}}, m_{1}, m_{2}$ being the masses of threshold states, and $B_{\infty}$ the infinite volume binding energy. Since here $m_{1}, m_{2}$ are masses of two baryons with multiple heavy quarks, $\Delta_{\mathrm{FV}}$ receives a large suppression even when $B_{\infty}$ is of a few MeV. This assures that the binding energies for these dibaryons, particularly for $\mathcal{D}_{b c}$, will be very close to the extracted mass differences that we showed in Fig. 1. Since we use the strange, charm, and bottom quark masses already at their physical values, the extracted mass differences, after assuming negligible finite volume corrections, are indeed the physical binding energies of these dibaryons. However, to confirm the bound state nature of these dibaryons, particularly for $\mathcal{D}_{b s}$ and $\mathcal{D}_{c s}$, one needs to carry out a detail finite volume study [49] and we will address that in the future.

For dibaryons, $\mathcal{D}_{c u}$ and $\mathcal{D}_{b u}$ we first perform chiral extrapolations with a constant plus a term linear in $m_{\pi}^{2}$.
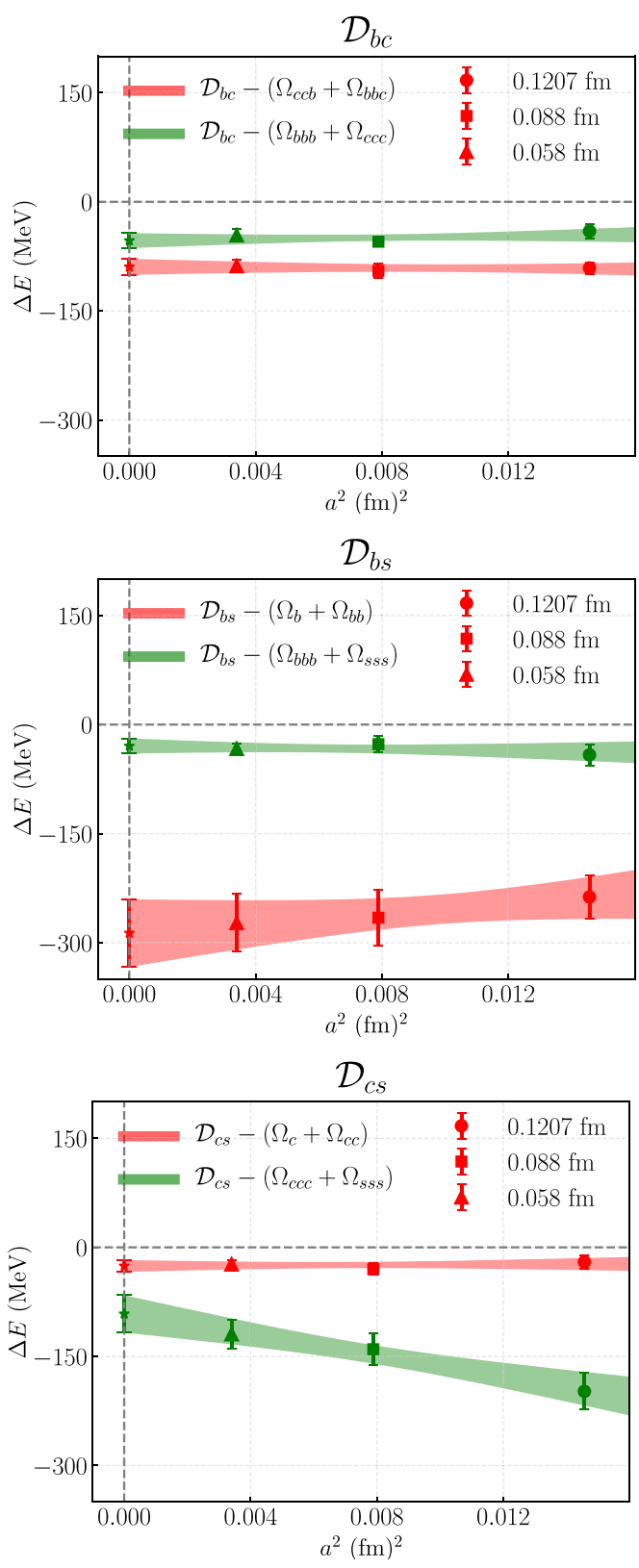

FIG. 1. Mass differences between various spin-1 heavy dibaryons $\left(\mathcal{D}_{q_{1} q_{2}}\right)$ from their two-baryon threshold states. Red points represent mass differences when the threshold states are with spin- $1 / 2$ baryons and the green points are the same with spin-3/2 baryons. Results are shown at three lattice spacings and at the continuum limit with shaded bands as one sigma error.

Because of the presence of light quarks, the signal-to-noise ratios in the correlation functions of these states are rather poor. Moreover for $\mathcal{D}_{b u}$, one decay product is the resonance state $\Delta_{\text {uиu }}$, which needs to be addressed with adequate finite volume study [49]. With the current lattice setup, it is therefore difficult for us to make a precise conclusive statement about the stability of these two dibaryons. In the future, such a study can be carried out with the availability of more computing resources. However, following the 
TABLE II. Energy differences between spin-1 heavy dibaryons and their two-baryon thresholds. The third column shows the predictions of masses for the stable dibaryons. The bottom part of the table is for dibaryons with unphysical quark $q$ with its mass $\left(m_{q}\right)$ varying in between the charm and 5 times the bottom quark masses. The errors within brackets incorporate both statistical and systematic errors added in quadrature.

\begin{tabular}{lccr}
\hline \hline & $\begin{array}{c}\text { Energy difference } \\
\text { from spin-1/2 }\end{array}$ & $\begin{array}{c}\text { Energy difference } \\
\text { from spin-3/2 }\end{array}$ & \multicolumn{1}{c}{ Mass } \\
Dibaryon & threshold [MeV] & threshold [MeV] & {$[\mathrm{MeV}]$} \\
\hline $\mathcal{D}_{b c}$ & $-91(12)$ & $-52(13)$ & $19105(21)$ \\
$\mathcal{D}_{b s}$ & $-287(45)$ & $-29(13)$ & $16004(17)$ \\
$\mathcal{D}_{c s}$ & $-26(9)$ & $-90(20)$ & $6381(20)$ \\
$\mathcal{D}_{b u}$ & $-350(110)$ & $3(50)$ & \\
$\mathcal{D}_{c u}$ & $-8(17)$ & $-75(46)$ & \\
$\mathcal{D}_{b q\left(m_{q}=1.38 m_{c}\right)}$ & & $-60(10)$ & \\
$\mathcal{D}_{b q\left(m_{q}=1.72 m_{c}\right)}$ & & $-87(8)$ & \\
$\mathcal{D}_{b q\left(m_{q}=2.07 m_{c}\right)}$ & & $-101(8)$ & \\
$\mathcal{D}_{b q\left(m_{q}=m_{b}\right)}$ & & $-109(5)$ & \\
$\mathcal{D}_{b q\left(m_{q}=2 m_{b}\right)}$ & & $-85(10)$ \\
$\mathcal{D}_{b q\left(m_{q}=5 m_{b}\right)}$ & & $-15(8)$ & \\
\hline \hline
\end{tabular}

example of the deuteron, if we assume they are also bound, our results suggest that their binding energies will be much smaller compared to the other three dibaryons mentioned above. The final values of the dibaryon masses are calculated by adding the known two-baryon threshold masses to the continuum value of binding energies that we extracted. For masses of yet-to-be discovered baryons we use their lattice values as calculated in this work and in Refs. $[32,33,47]$. We also use the subtraction method $[32,33,47,51]$ utilizing the spin-average value of $1 S$ charmonia and bottomonia and find results consistent with the above mass estimates. The final values of the dibaryon masses are shown in the third column of Table II. In Fig. 2 we show the relative energy levels of these dibaryons with respect to their spin-1/2 and spin-3/2 two-baryon

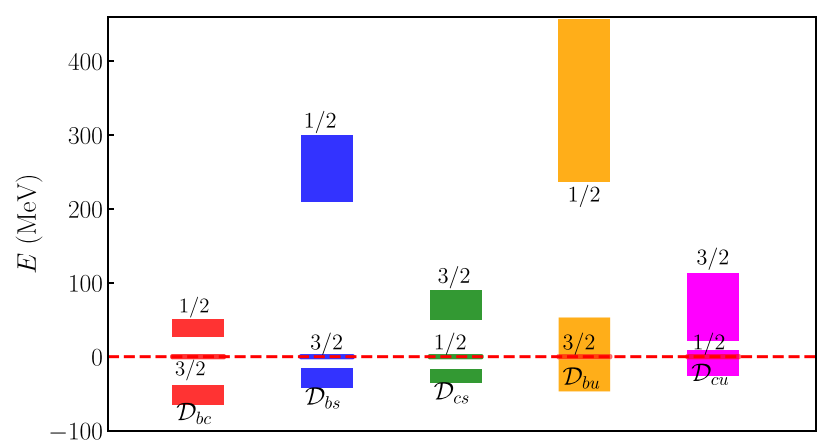

FIG. 2. Relative energy levels of the spin-1 dibaryons and their respective two-baryon noninteracting strong-decay threshold states. The horizontal line is for the closest threshold (normalized to zero) while $1 / 2$ and $3 / 2$ signify the spin of the constituent single baryons of the two-baryon threshold. noninteracting strong-decay thresholds. It is quite apparent that the dibaryons $\mathcal{D}_{b c}, \mathcal{D}_{b s}$, and $\mathcal{D}_{c s}$ lie below their respective closest thresholds (horizontal zero line) by about 4,2 , and 3 sigma errors, respectively, while for the other two, $\mathcal{D}_{b u}$ and $\mathcal{D}_{b u}$, we could not reach a definitive conclusion due to large errors. It may be noted here that we have obtained our results from simulations with a finite number of statistical ensembles, and on space-time grids with finite lattice spacings and finite volume, and hence these are associated with both statistical and systematic errors. Our final results are obtained after carefully addressing those errors and we elaborate on that in the Supplemental Material [45].

Interestingly, our results point out that the strong binding energies of these heavy dibaryons increase as they become heavier in mass. To confirm this pattern we also calculate similar dibaryons, $\mathcal{D}_{b q}$, with bottom quark at its physical value while varying the other quark mass $\left(m_{q}\right)$ between the charm to five times the bottom quark masses (for $m_{q}>m_{b}$, we use the same tuning coefficients as for $m_{q}=m_{b}$ ). Of course, such quarks are unphysical and so are these dibaryons, but since they obey the same strong dynamics they can clarify the pattern of stability. In Table II, at the bottom part, we tabulate the binding energies of these unphysical dibaryons (these results are obtained only at our finest lattice).

To depict it more clearly, in Fig. 3 we plot these binding energies for dibaryons $\mathcal{D}_{b q}\left(\Omega_{b b b} \Omega_{q q q}\right)$ with $q$ varying from light to all the way to the bottom quark. It clearly shows that the spin-1 dibaryons become more stable when they are heavier. We even calculate such dibaryons with much higher quark masses (which are of course unphysical) and observe that their binding energies decrease when $m_{q}>m_{b}$ and they vanish at very large quark masses $\left(m_{q} \rightarrow \infty\right)$. This suggests that the combination $\mathcal{D}_{b q}\left(\Omega_{b b b} \Omega_{q q q, q=b}\right)$ has the maximum binding.

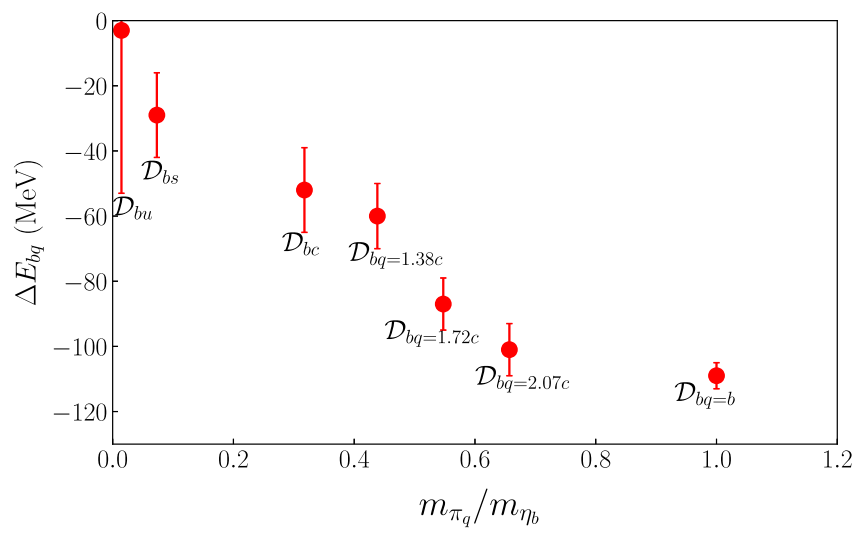

FIG. 3. Binding energy of the spin-1 dibaryon $\mathcal{D}_{b q}$ where we kept the bottom quark fixed and vary the other quark $q$ from light to bottom quarks. Along the $x$ axis we show the ratio of the pseudoscalar mass at $m_{q}\left(m_{\pi_{q}}\right)$ to that at the bottom quark $\left(m_{\eta_{b}}\right)$. As the mass of the quark $q$ increases $\left(m_{q}=m_{u}\right.$ to $\left.m_{b}\right)$, the dibaryon binding energy also increases. 
The strong binding of these dibaryons and the pattern that it increases with the increase of quark mass is pointing towards an interesting strong dynamics in the heavy hadrons. It is to be noted that for the doubly heavy tetraquarks, lattice studies [19,21-23] found a complete opposite patterns where the strong binding increases as the light quark becomes lighter. This suggests that the strongbinding mechanisms of spin-1 heavy tetraquarks and spin-1 heavy dibaryons are different and certainly this needs to be investigated further, perhaps similarly to the way it was investigated in Ref. [22] for the heavy tetraquarks.

The stability of these dibaryons against the strong and electromagnetic decays opens up the possibility of finding these and even more complicated higher nuclei with many heavy quarks, similar to nuclei of various elements in our periodic table. Similar to the role of the deuteron in the nuclear fusion cycle for the creation of elements, these dibaryons can help the fusion processes of heavy baryons to produce nuclei with heavy quarks. Such nuclei can be studied theoretically in the future with adequate computational resources and it may well be possible to discover them in future higher energy heavy ion facilities. It will also be interesting to see if the formation of such states has any implication in cosmology. Formation of such hadrons also enhances the speculation on the possibility of a quark-level analog of nuclear fusion which was discussed recently in Ref. [10]. For example, formation of $\mathcal{D}_{b s}$ through fusion of $\Omega_{b b}$ and $\Omega_{b}$, as well as through fusion of $\Omega_{b b b}$ and $\Omega_{s s s}$, are highly exothermic with the release of energy about 300 and $30 \mathrm{MeV}$, respectively. We also find that reactions such as $\mathcal{B}_{q b b}^{1 / 2}+\mathcal{B}_{q q b}^{1 / 2} \rightarrow \mathcal{B}_{b b b}^{3 / 2}+\mathcal{B}_{q q q}^{3 / 2}, q \equiv c, s, u / d$ and $\mathcal{B}_{c c c}^{3 / 2}+$ $\mathcal{B}_{q q q}^{3 / 2} \rightarrow \mathcal{B}_{q c c}^{1 / 2}+\mathcal{B}_{q q c}^{1 / 2}, q \equiv s, u / d$, are highly exothermic (here we represent $\mathcal{B}^{J} q_{1} q_{2} q_{3}$ as a baryon with spin $J$ and quark contents $q_{1}, q_{2}$, and $q_{3}$ ).

Since these dibaryons involve quarks with masses over a wide range, studies of their properties will be helpful to understand the strong dynamics at multiple scales. Because of the presence of multiple heavy quarks they will decay via various possible weak decay processes. For example, $\mathcal{D}_{c b}$ can decay through $b \rightarrow c, b \rightarrow s$, and $c \rightarrow s$ to various light baryons and multiple mesons, which can interfere among themselves. Detailed analysis of these multiple ways of decay may also be an ideal place to study hadronic interference in weak decay processes. Furthermore, this work, particularly the strong binding of heavier dibaryons, motivates us to study other heavy dibaryons, namely, spin-0 states as well as heavy quark analogs of the $\mathrm{H}$ dibaryon. Results on those dibaryons will be reported in the future which, taken together with the findings here, may well be able to infer important information on the dynamics and binding mechanisms of heavy hadrons and perhaps also for the nuclei with heavy quarks.

Computations were carried out on the Cray-XC30 of ILGTI, TIFR, and on the Gaggle/Pride clusters of the
Department of Theoretical Physics, TIFR. We would like to thank A. Dighe, M. Padmanath, S. Pavaskar, and S. Raychaudhuri for many useful discussions. We are thankful to the MILC Collaboration and in particular to S. Gottlieb for providing us with the HISQ lattices. N. M. would also like to thank A. Salve, K. Ghadiali, and P. M. Kulkarni for computational support.

*parikshit@theory.tifr.res.in nilmani@theory.tifr.res.in

[1] R. L. Jaffe, Phys. Rev. D 15, 267 (1977).

[2] P. J. Mulders, A. T. Aerts, and J. J. de Swart, Phys. Rev. D 21, 2653 (1980).

[3] A. P. Balachandran, A. Barducci, F. Lizzi, V. G. J. Rodgers, and A. Stern, Phys. Rev. Lett. 52, 887 (1984).

[4] T. Sakai, K. Shimizu, and K. Yazaki, Prog. Theor. Phys. Suppl. 137, 121 (2000).

[5] Y. Ikeda and T. Sato, Phys. Rev. C 76, 035203 (2007).

[6] M. Bashkanov, S. J. Brodsky, and H. Clement, Phys. Lett. B 727, 438 (2013).

[7] P. E. Shanahan, A. W. Thomas, and R. D. Young, Phys. Rev. Lett. 107, 092004 (2011).

[8] H. Clement, Prog. Part. Nucl. Phys. 93, 195 (2017).

[9] J. P. Lees et al. (BABAR Collaboration), Phys. Rev. Lett. 122, 072002 (2019).

[10] M. Karliner and J. L. Rosner, Nature (London) 551, 89 (2017).

[11] M. Tanabashi et al. (Particle Data Group), Phys. Rev. D 98, 030001 (2018).

[12] S. K. Choi et al. (Belle Collaboration), Lepton and photon interactions at high energies, Proceedings of the $23 \mathrm{rd}$ International Symposium, LP2007, Daegu, South Korea, 2007; Phys. Rev. Lett. 100, 142001 (2008).

[13] R. Aaij et al. (LHCb Collaboration), Phys. Rev. Lett. 112, 222002 (2014).

[14] A. Bondar et al. (Belle Collaboration), Phys. Rev. Lett. 108, 122001 (2012).

[15] R. Aaij et al. (LHCb Collaboration), Phys. Rev. Lett. 115, 072001 (2015).

[16] R. Aaij et al. (LHCb Collaboration), Phys. Rev. Lett. 122, 222001 (2019).

[17] M. Karliner and J. L. Rosner, Phys. Rev. Lett. 119, 202001 (2017).

[18] E. J. Eichten and C. Quigg, Phys. Rev. Lett. 119, 202002 (2017).

[19] A. Francis, R. J. Hudspith, R. Lewis, and K. Maltman, Phys. Rev. Lett. 118, 142001 (2017).

[20] P. Bicudo, M. Cardoso, A. Peters, M. Pflaumer, and M. Wagner, Phys. Rev. D 96, 054510 (2017).

[21] P. Junnarkar, N. Mathur, and M. Padmanath, Phys. Rev. D 99, 034507 (2019).

[22] A. Francis, R. J. Hudspith, R. Lewis, and K. Maltman, Phys. Rev. D 99, 054505 (2019).

[23] L. Leskovec, S. Meinel, M. Pflaumer, and M. Wagner, Phys. Rev. D 100, 014503 (2019).

[24] T. Inoue, N. Ishii, S. Aoki, T. Doi, T. Hatsuda, Y. Ikeda, K. Murano, H. Nemura, and K. Sasaki (HAL QCD Collaboration), Phys. Rev. Lett. 106, 162002 (2011). 
[25] S. R. Beane et al. (NPLQCD Collaboration), Phys. Rev. Lett. 106, 162001 (2011).

[26] S. R. Beane, E. Chang, W. Detmold, H. W. Lin, T. C. Luu, K. Orginos, A. Parreno, M. J. Savage, A. Torok, and A. Walker-Loud (NPLQCD Collaboration), Phys. Rev. D 85, 054511 (2012).

[27] T. Yamazaki, K.-i. Ishikawa, Y. Kuramashi, and A. Ukawa, Phys. Rev. D 86, 074514 (2012).

[28] S. Gongyo et al., Phys. Rev. Lett. 120, 212001 (2018).

[29] A. Francis, J. R. Green, P. M. Junnarkar, C. Miao, T. D. Rae, and H. Wittig, Phys. Rev. D 99, 074505 (2019).

[30] T. Iritani et al., Phys. Lett. B 792, 284 (2019).

[31] S. Basak, S. Datta, M. Padmanath, P. Majumdar, and N. Mathur, Proceedings of the 30th International Symposium on Lattice Field Theory (Lattice 2012), Cairns, Australia (2012); Proc. Sci., LATTICE2012 (2012) 141.

[32] N. Mathur, M. Padmanath, and S. Mondal, Phys. Rev. Lett. 121, 202002 (2018).

[33] N. Mathur and M. Padmanath, Phys. Rev. D 99, 031501(R) (2019).

[34] A. Bazavov et al. (MILC Collaboration), Phys. Rev. D 87, 054505 (2013).

[35] E. Follana, Q. Mason, C. Davies, K. Hornbostel, G. P. Lepage, J. Shigemitsu, H. Trottier, and K. Wong (HPQCD and UKQCD Collaborations), Phys. Rev. D 75, 054502 (2007).

[36] A. Bazavov et al. (MILC Collaboration), Phys. Rev. D 93, 094510 (2016).

[37] H. Neuberger, Phys. Lett. B 417, 141 (1998).

[38] H. Neuberger, Phys. Lett. B 427, 353 (1998).

[39] M. Luscher, Phys. Lett. B 428, 342 (1998).

[40] G. P. Lepage, L. Magnea, C. Nakhleh, U. Magnea, and K. Hornbostel, Phys. Rev. D 46, 4052 (1992).

[41] R. J. Dowdall et al. (HPQCD Collaboration), Phys. Rev. D 85, 054509 (2012).

[42] A. X. El-Khadra, A. S. Kronfeld, and P. B. Mackenzie, Phys. Rev. D 55, 3933 (1997).

[43] B. Chakraborty, C. T. H. Davies, B. Galloway, P. Knecht, J. Koponen, G. C. Donald, R. J. Dowdall, G. P. Lepage, and C. McNeile, Phys. Rev. D 91, 054508 (2015).
[44] K. G. Wilson, Phys. Rev. D 10, 2445 (1974).

[45] See Supplemental Material at http://link.aps.org/ supplemental/10.1103/PhysRevLett.123.162003 which provide the procedure that we utilize for computing energy differences between the ground state dibaryons from their non-interacting two-baryon thresholds. In the second part we also address statistical and systematic errors associated with this calculation, which includes Refs. [32,33,36, 42,43,46-53].

[46] G. P. Lepage, B. Clark, C. T. H. Davies, K. Hornbostel, P. B. Mackenzie, C. Morningstar, and H. Trottier, Lattice field theory, Proceedings of the 19th International Symposium, Lattice 2001, Berlin, Germany (2001); Nucl. Phys. B, Proc. Suppl. 106-107, 12 (2002).

[47] Z. S. Brown, W. Detmold, S. Meinel, and K. Orginos, Phys. Rev. D 90, 094507 (2014).

[48] S. Basak, S. Datta, A. T. Lytle, M. Padmanath, P. Majumdar, and N. Mathur, Proceedings of the 31st International Symposium on Lattice Field Theory (Lattice 2013), Mainz, Germany, 2013; Proc. Sci., LATTICE2013 (2014) 243.

[49] M. Luscher and U. Wolff, Nucl. Phys. B339, 222 (1990).

[50] C. McNeile, C. T. H. Davies, E. Follana, K. Hornbostel, and G. P. Lepage, Phys. Rev. D 86, 074503 (2012).

[51] R. J. Dowdall, C. T. H. Davies, T. C. Hammant, and R. R. Horgan, Phys. Rev. D 86, 094510 (2012).

[52] S. Basak, S. Datta, N. Mathur, A. T. Lytle, P. Majumdar, and M. Padmanath (ILGTI Collaboration), Proceedings of the 32nd International Symposium on Lattice Field Theory (Lattice 2014), Brookhaven, NY, USA (2014); Proc. Sci., LATTICE2014 (2015) 083.

[53] S. Borsanyi et al., Science 347, 1452 (2015).

[54] S. R. Beane, P. F. Bedaque, A. Parreno, and M. J. Savage, Phys. Lett. B 585, 106 (2004).

[55] Z. Davoudi and M. J. Savage, Phys. Rev. D 84, 114502 (2011).

[56] R. A. Briceno, Z. Davoudi, T. Luu, and M. J. Savage, Phys. Rev. D 88, 114507 (2013). 